\title{
Estudo da viabilidade técnica de implantação de aerogerador de pequeno porte de energia elétrica renovável no Estado de Alagoas
}

\section{Luiz André da Silva Lima*, Valéria Loureiro da Silva}

Faculdade SENAI CIMATEC. Av. Orlando Gomes, 1845. Piatã. Salvador-BA (CEP 41650-010). E-mail: andre.lima@al.senai.br.

Resumo. O presente trabalho descreve a análise dos parâmetros das características eficiência de faixa de regime de trabalho de um aerogerador de pequeno porte do modelo ISTA-BREEZE 500, de fabricação turca, eólico, que inicialmente gera entre 400 a $500 \mathrm{~W}$ e tensão de saída $24 \mathrm{v}$, peso $2,5 \mathrm{~kg}$, na cor branca, velocidade de pá de $2,5 \mathrm{~m} / \mathrm{s}$. A partir destas informações foi desenvolvido um estua de viabilidade técnica de um aerogerador de pequeno porte de ene sia elétrica. Os resultados obtidos foram realizados em uma bancada qual tiveram dados satisfatórios nos três testes apresentad ora For realizados os teste de tensões de saída do aerogerador m

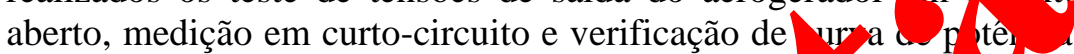
O laboratório do Centro de Formação Profissior Gustar aiva (CFP-GP), SENAI, Maceió-AL, Brasil, foi o local de u A bancada mostrou ser apropriada para estes tipos en ser utilizada para analisar a viabilidade técr a plantação de aerogerador de pequeno porte de energia trica on vavel.

Palavras-chaves: Aerogerador Fonte nnativa; Fontes renováveis de energia; Energia eólica.

Abstract. Study of the $\mathrm{Cr}$ al bility of implementation of aircraft of small energ. enewable energy. The present work describes the an sis parameters of the efficiency characterist ${ }^{-}$of th operating range of a small wind turbine of the ISTA-BREE of Turkish, wind turbine manufacturing, that initially ger es 400 to $500 \mathrm{~W}$ and output voltage $24 \mathrm{v}$, weight $2.5 \mathrm{~kg}$, white, sta velocity $2.5 \mathrm{~m} / \mathrm{s}$. From this information was developed a technikal feasibility study of a small electric power generator. The results were obtained in a bench, in which they had satisfactory data in the three tests presented. The open-circuit aerogenerator output voltages test, short-circuit measurement and power curve verification were performed. The laboratory of the Gustavo Paiva Vocational Training Center (CFP-GP), SENAI, at Maceió-AL, Brazil, was the test site. The workbench proved to be appropriate for these types of tests and could be used to analyze the technical viability of small wind turbine implantation.

Keywords: Wind turbine; Alternative source; Renewable sources of energy; Wind energy. 


\section{Introdução}

Uma das primeiras estratégias empregadas pelo homem para utilizar a força dos ventos foi através dos barcos à vela. Com a utilização dos moinhos de grãos e aparatos de bombeamento de água, o objetivo era diminuir todos os esforços físicos. O primeiro moinho de vento foi movido pela força humana. Escravos e também alguns animais eram usados para fazê-lo girar em círculos. Os moinhos em poste ou pilastra, os seus mancais, eram parte do capitel (parte superior) que segurava as lâminas. Os trabalhadores tiveram que direcionar sua máquina de vento até a invenção da ventoinha. Após o ano de 1750, o sistema ficou automático, direcionado e acionado pelo próprio vento (Martins et al., 2008). Os primeiros moinhos de vento eram de eixo vertical, como o mostrado na Figura 1a, e depois de eixo horizontal (Matos, 2009), como na Figura 1b. sendo aproveitadas para suprir a necessidade de energia adicional no país. Ela também não é suficiente, precisando de outros tipos de fontes para suprir a necessidade do Brasil. As renováveis são as energias das ondas e marés, energia solar, energia hidráulica, que é a mais utilizada no Brasil, e energia eólica (Reis e Lineu Belico, 2015).

A energia eólica utiliza a força dos ventos para virar as hélices para a geração de energia, Ela vem crescendo a cada ano, podendo ser considerada uma das promissoras fontes de energia natural no nosso país.

Os aerogerador s 4 equeno porte (APP) estão n recent tran rmações do setor de geraçâo trib oro novido pela

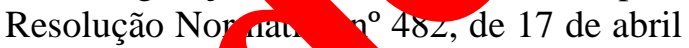
de 2012, da ência ćional de Energia Elétrica (AN ) O Programa de Incent vo Vontes Alternativas de Energia Elétri ( $\mathrm{h} / \mathrm{FA})$ do governo federal o ob 0 de aumentar a participação du $\mathrm{VE}$ produzida por empreendimentos con os com base na energia eólica \begin{tabular}{|c|c|c|}
\hline Eixo Vertical & Eixo Horizontal biomassa e pequenas centrais \\
\hline & 1 & elétricas (PCH) no Sistema Elétrico \\
nterligado Nacional (SIN) (Salino, 2011).
\end{tabular}

O intuito do PROINFA é promover a diversificação da Matriz Energética Brasileira como fonte alternativa de aumento com segurança do abastecimento de energia elétrica, valorizando potencialidades regionais e locais do território nacional (NEOENERGIA, 2009).

No Brasil, algumas normas da Comissão Eletrotécnica Internacional (IEC) sobre EE foram traduzidas e denominadas NBR IEC 61400-1, NBR IEC 61400-12-1. A IEC 61400-2 refere-se a aerogeradores de pequeno porte. $\mathrm{O}$ mercado de aerogeradores de grande porte tem se tornado significativo, principalmente em função dos leilões de energia de reserva e de FE renováveis. Todavia, a comercialização no mercado de APP no país não tem muita procura no mercado brasileiro, é praticamente inexistente, uma vez que a geração de energia elétrica no Brasil é predominantemente centralizada (Vieira, 2013). Uma das mais utilizadas, entre as ñ̃ renováveis, é a termoelétricas, que vem 
Tabela 1. Fabricantes de aerogeradores de pequeno porte.

\begin{tabular}{|c|c|c|c|c|c|}
\hline $\begin{array}{c}\text { Fabricante / modelo } \\
\text { do aerogerador }\end{array}$ & $\begin{array}{l}\text { Tensão de } \\
\text { saída }\end{array}$ & $\begin{array}{l}\text { Potência } \\
\text { (W) }\end{array}$ & $\mathrm{rpm} / \mathrm{m} / \mathrm{s}$ & $\begin{array}{c}\text { Outras } \\
\text { característica }\end{array}$ & $\begin{array}{c}\text { Valor do } \\
\text { aerogerador } \\
\text { (R\$) } \\
\end{array}$ \\
\hline $\begin{array}{l}\text { Enersud LTDA / } \\
\text { Notus } 138\end{array}$ & $\begin{array}{l}\text { 12/24/48 } \\
\text { Sistema } \\
\text { Trifásico }\end{array}$ & $250-350$ & $\begin{array}{c}1.100 / \\
(3-12 \mathrm{~m} / \mathrm{s})\end{array}$ & $\begin{array}{l}\text { Números de pás: } 5 \\
\text { Tipo de pás: torcida } \\
\text { Alternador: fluxo } \\
\text { axial }\end{array}$ & $3.500,00$ \\
\hline $\begin{array}{l}\text { Eletrovento / } \\
\text { Turbo } 500\end{array}$ & $\begin{array}{l}\text { 12V / Sistema } \\
\text { Trifásico }\end{array}$ & $400-500$ & $\begin{array}{c}600-1000 / \\
(3-12 \mathrm{~m} / \mathrm{s})\end{array}$ & Números de pás: 3 & $2.867,39$ \\
\hline $\begin{array}{l}\text { ISTA BREEZE / } \\
50024 \mathrm{v}\end{array}$ & $24 \mathrm{v}$ & $400-500$ & $\begin{array}{c}650-3000 / \\
12 \mathrm{~m} / \mathrm{s}\end{array}$ & Números de pás: 3 & $1.650,00$ \\
\hline
\end{tabular}

\section{A Figura 2 mostra} esquematicamente um sistema isolado de geração de energia eólica. Consiste de um controlador de carga que monitora a carga gerada pelo aerogerador e a armazena nas baterias, onde sua principal função é bloquear a corrente reversa e prevenir a sobrecarga das baterias, prevenir o excesso de descarga e sinalizar o estado da bateria e fluxo de carga através de mostrador (Matos, 2009).

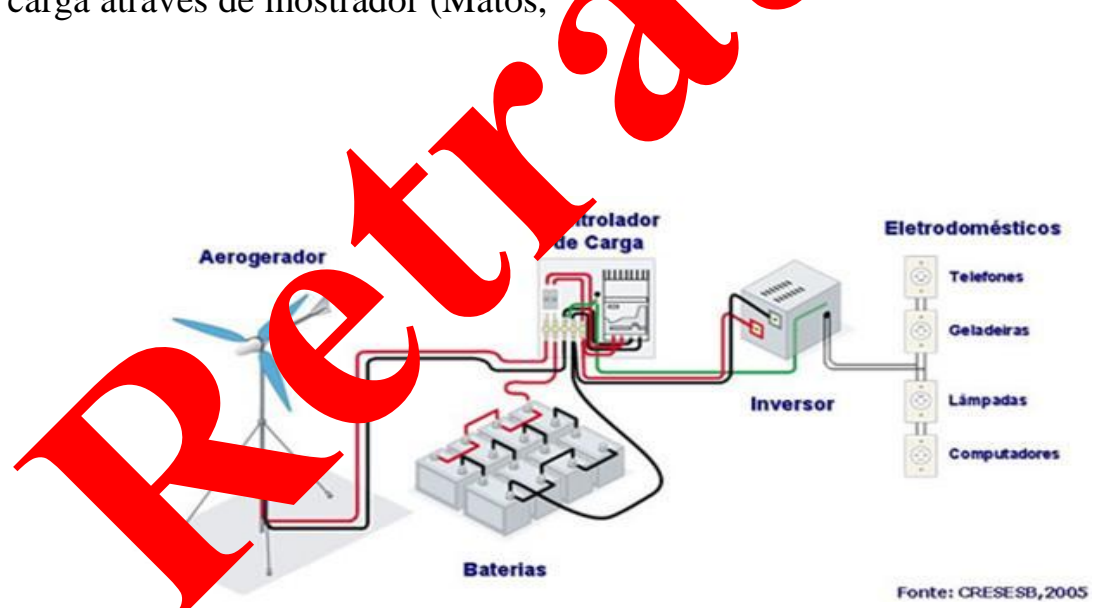

Figura 2. Configuraçáo de um sistema eólico isolado mostrando o aerogerador, controlador de carga, bateria e inversor.

Este trabalho propõe analisar um aerogerador existente em uma bancada e, a partir desta análise, desenvolver um estudo de viabilidade técnica de um APP de energia elétrica para regiões do nordeste, especificamente no estado de Alagoas. De acordo com o atlas Eólico, foi feita uma análise mostrando que o Estado de Alagoas tem um considerável potencial eólico. A região mais propícia do estado seria o agreste, em seguida do sertão. O objetivo do trabalho é fazer um levantamento do gráfico de desempenho de um AEPP em uma bancada. Podemos testar vários AEPP permitindo fazer a comparação dos resultados práticos com os resultados 
teóricos feitos com a do fabricante (Ferreira Junior e Rodrigues, 2015).

\section{Metodologia}

Para avaliar a viabilidade técnica de utilizar um aerogerador eólico de pequeno porte (AEPP), foi montada uma bancada de testes, conforme a Figura 3.

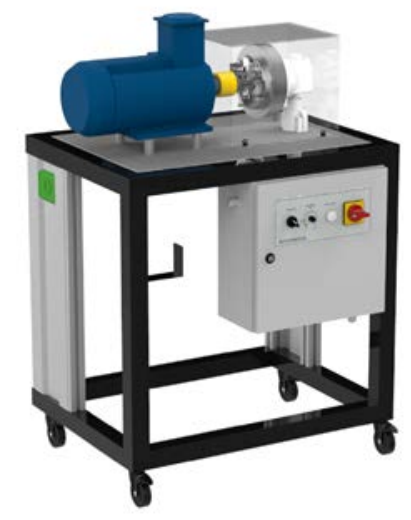

Figura 3. Módulo eólico com simulador.

Teste

\section{Equipamento da Bancada}

A bancada é compo inversor de frequência (1), m elétri (2), torquímetro (3), aernoera (4), multímetro (5), retificad or fásico (6), controlador do gado 4 , iny ersor ou converso de tens DC 8), duas baterias est naria $15 \mathrm{Ah} / 12$ Vcc ligada em série (9), (ro,. gura 4 mostra o esquema de funo mento da bancada.

\section{Aerogerador estudado}

$O$ aerogerador que foi utilizado é do fabricante ISTA-BREEZE modelo 500, de fabricação turca, eólico, que inicialmente gera 400 a $500 \mathrm{~W}$ e tensão de saída $24 \mathrm{~V}$, peso $2,5 \mathrm{~kg}$, na cor branca, velocidade de partida de $2,5 \mathrm{~m} / \mathrm{s}$, sua velocidade média mensal do vento a $5,4 \mathrm{~m} / \mathrm{s}(19,44 \mathrm{~km} / \mathrm{h})$ : $150 \mathrm{kwh} / \mathrm{mês}$. Sua estrutura externa é feita de Resina de Poliéster Termoplástico Crastin ${ }^{\circledR}$ PBT, reforçado com $30 \%$ de fibra de vidro, as lâminas do rotor e o invólucro são altamente duráveis. O contato livre de manutenção, deslizante (sem carbono), garante um bom fluxo de corrente e, portanto, nenhuma torção do cabo no mastro. Outras características técnicas posteriores na descrição. Conforme a Figura 5.

Teste analisados no Aerogerador

Todos os testes para analisar os parâmetros do aerogerador de pequeno porte foram realizados na bancada, dentro do laboratório do Centro de Formação Profissional Gustavo Paiva (CFP-GP) SENAI, na Rua Pedro Américo, $n^{\circ}$. 18, Poço, Maceió - Alagoas. Para simular as condições reais do vento, foi utilizado um inversor de frequênci controlar a velocidade do otor fásic íncrono de $1 \mathrm{CV}$ e com 180 M, mo or acoplado diretamente ao $\mathrm{cIX}_{\mathrm{u}}$ aerogerador que será testado, confo a a 4. No eixo do aerogerador fo ned ado um torquímetro para f Zel vediçau do torque no mesmo. Ao Oes th tempo, foi conectado trumer e medição como o multímetro e icate amperímetro para fazer as mea. de tensão e corrente por cada fase coletar dados das características nominais aerogerador, em várias situações, tais omo:

- $\quad$ Tensões de saída do aerogerador em circuito aberto;

- $\quad$ Medição em curto-circuito;

- Verificação de curva de potência segundo IEC 61400-12-1;

\section{Procedimento de medição do instrumetro}

Para fazer o primeiro teste, em circuito aberto, foi utilizado o aerogerador descrito no item [2.2]. Sua medição foi feita na saída do aerogerador, dada em tensão CA, constituídas de três bobinas com defasagem angular de $120^{\circ}$. Como ele tem três fases, fase $\mathrm{A}, \mathrm{B}$ e $\mathrm{C}$, medimos entre a fase $\mathrm{A}$ e $\mathrm{B}$, depois entre $\mathrm{A}$ e $\mathrm{C}$, e, em seguida $\mathrm{B}$ e $\mathrm{C}$. Essa tensão foi medida nos terminais de saída do aerogerador e chamada de tensão de linha.

Foi acoplado no seu eixo um tacômetro, que é um equipamento utilizado para medir rotação do motor. A curva também foi construída através da TENSÃO 
versus RPM e calculada com os dados de tensão e RPM medidos durante a operação do aerogerador. Com a análise, foi mostrado que para quando aumentava o valor do RPM, os valores de tensão do terminais de saída do aerogeradores foram aumentados e tinha perda mecânica, para fazer a medição do circuito aberto.

As medições em curto-circuito foram coletadas através de simulação na bancada, colocando o multímetro na escala de corrente em cada fase, na A,B e C, nas saída dos terminais do aerogerador as fase foram conectados entre si, fechando um curto-circuito. Foram feitas as medições de corrente e o torque para cada faixa de velocidade. O equipamento utilizado para medir a rotação do motor é o tacômetro, conforme Figura 4.

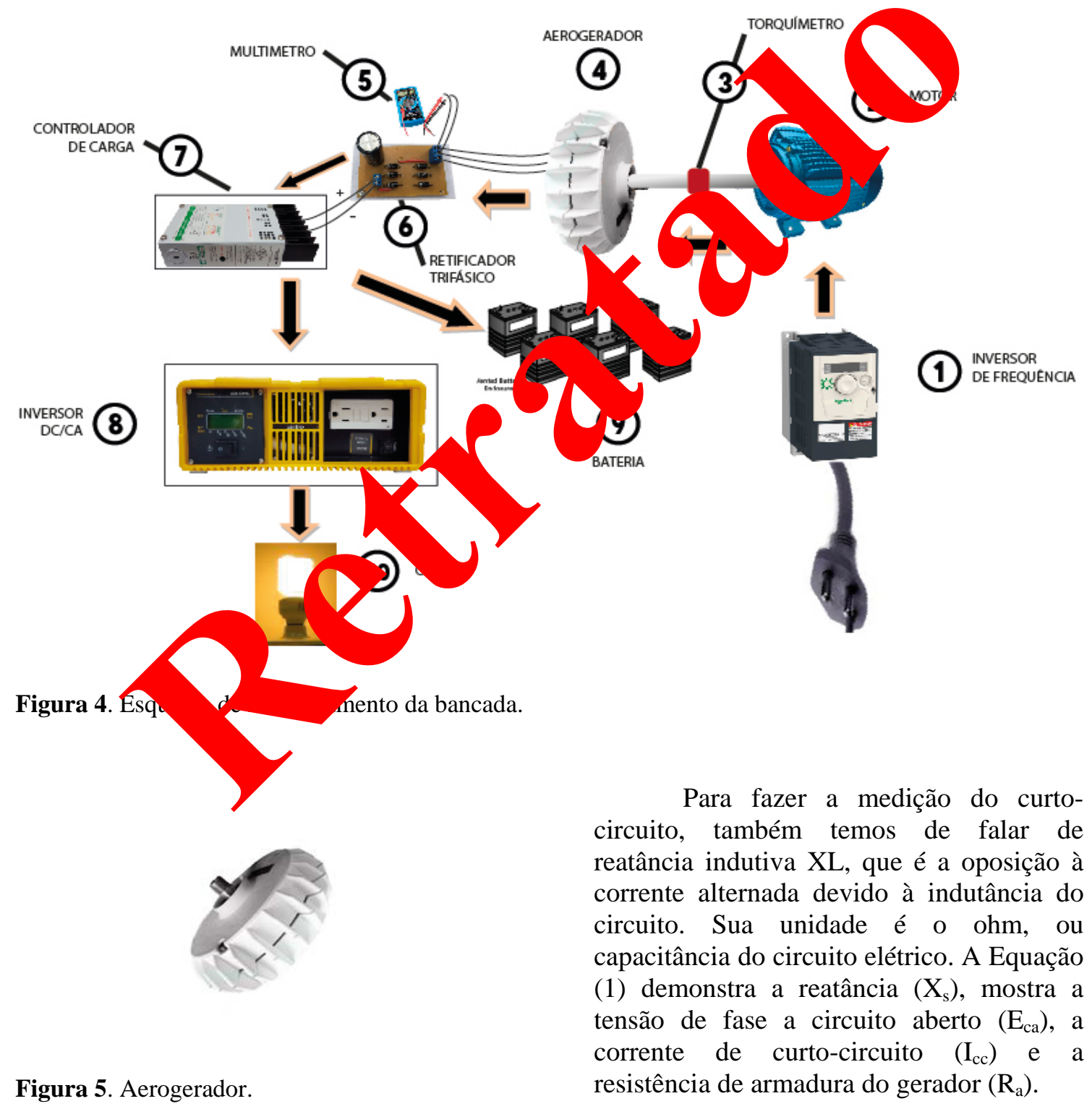

Rev. Bras. Gest. Amb. Sustent., 2016, v. 3, n. 6, p. 307-316. 


$$
X_{\mathrm{s}}=\sqrt{\left(\mathrm{E}_{\mathrm{ca}} / \mathrm{I}_{\mathrm{cc}}\right)^{2}-\mathrm{R}_{\mathrm{a}}{ }^{2}}
$$

Para medir a curva de potência e para realizar as medições de valor da potencia foi feita a medição de corrente, tensão de saída dos terminais do aerogerador e torque.

Estudo do potência do vento em função da velocidade do vento

Para fazer a simulação da velocidade do vento, os experimentos foram feitos na bancadas, utilizando velocidade inicial de 1 RPM em ordem crescente até 1.150 RPM. Para poder avaliar qual seria seu valor de tensão, corrente e potência, foi colocado um valor de rotação e verificado os valores de todas as grandezas citadas, para a construção da Figura 6 que mostra o ensaio do circuito aberto, Já para a Figura 7 e 8, os valores de rotação foram menores de 1 RPM até 900 RPM. Na Figura 7, o teste era para simular um curto-circuito entre as fases, e, na simulação da Figura 8, da curva de potência do aerogerador. Para geração de cada potência, foi colocado um valor de RPM. Através da simulação do aerogera mostramos um resumo dos resultado os dados coletados foram colocaros planilha do Excel, comparan com dados extraídos do catálogo do ricant para avaliar a viabilidade $d$ mo.

Com os dados tipos, podemos prever o dese $\mathrm{O}$ de pamento em outros lo is em le o mèsmo possa ser instalado. $\mathrm{a}$ ar se o litoral da região nora no estado de Alagoas é realmente viável. ơ sua velocidade média do vento é de ordem de $4 \mathrm{~m} / \mathrm{s}$ a $10 \mathrm{~m}$ de altura, vamos analisar a viabilidade da tecnologia eólica de pequeno porte "no Estado de Alagoas” (Costa, 2009).

Para calcular o potencial do vento ou potência eólica, temos que saber os valores da velocidade do vento, conforme a Fórmula 2.

$$
\mathrm{P}_{\text {vento }}=1 / 2 . \rho \cdot \mathrm{A} \cdot \mathrm{V}^{3}{ }_{\text {vento }} \mathrm{W} \text { atts }
$$

Onde:

P é a potência do vento (Watt); $\left.\mathrm{kg} / \mathrm{m}^{3}\right)$;

$\rho$ é a densidade do ar $(\rho=1,2256$

A área das pás $\left(\mathrm{m}^{2}\right),\left(\pi \mathrm{R}^{2}\right)$;

$\mathrm{V}_{\text {Vento }}$ é a velocidade do vento incidente na turbina eólica $(\mathrm{m} / \mathrm{s})$.

Para calcular a potência elétrica do aerogerador do vento ou potência eólica, temos quer saber os valores da velocidade do vento, conforme a fórmula 3 .

\section{$\left.\mathrm{kg} / \mathrm{m}^{3}\right)$;}

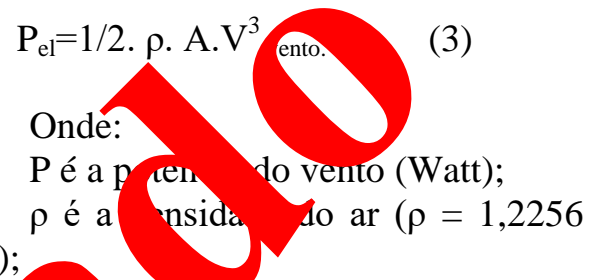

$\checkmark$ ento a velocidade do vento

idente arbina eólica (m/s).

Gp e o coeficiente de potência.

é a velocidade do vento incidente turrbina eólica $(\mathrm{m} / \mathrm{s})$.

Determinação da energia convertida por um aerogerador

Para calcular o intervalo de tempo durante o qual cada velocidade do vento ocorreu, é só multiplicar a função densidade de probabilidade de Weibull pelo número de horas do período considerado (Lopes e Almeida, 2003).

$$
N(x)=f(V) . t
$$

Onde:

$\mathrm{N}(\mathrm{x})$ é o intervalo de tempo durante o qual cada velocidade do vento ocorreu;

$\mathrm{f}(\mathrm{V})$ é a função densidade de probabilidade de Weibull;

t é o número de horas do período considerado. 
Cada fabricante de aerogerador fornece sua própria curva de potência e a curva da energia convertida em função da velocidade do vento $\mathrm{P}(\mathrm{V})$. Para saber qual é a curva da energia convertida pelo aerogerador é realizada a multiplicação da função densidade de probabilidade pelo número de horas do período considerado e pela curva de potência do aerogerador em análise, conforme a Fórmula 5 (Vale, 2000).

$$
E(V)=f(V) \cdot t \cdot P(V)
$$

$\mathrm{E}(\mathrm{V})$ é curva da energia convertida pelo aerogerador como uma função da velocidade do vento para certo período;

$\mathrm{f}(\mathrm{V})$ é a função densidade de probabilidade de Weibull;

t é o número de horas do período considerado;

$\mathrm{P}(\mathrm{V})$ é a curva de potência do aerogerador em análise.

A curva de potência poderá, ainda, ser determinada de forma experimental utilizando instrumento de medida, como o wattímetro, ou calculados através Fórmula 5, ou utilizando-se um multýn tro na escala de tensão e tambén amperímetro para medir a corı teremos a potência medições na saía de potin eólico. a corrente.

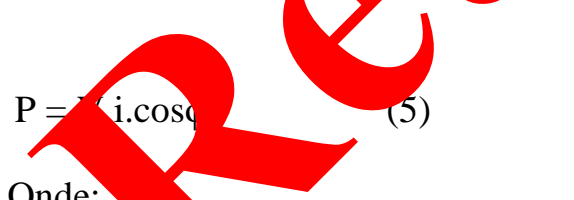

Onde:

$P$ é a potê calculada (em watt);

$\mathrm{V}$ é a tensão nedida (em volt);

i é a corrente medida (em ampère); $\varphi$ é o ângulo de fase entre a tensão e

\section{Resultados e discussão}

\section{Medição em circuito aberto}

Os dados da Figura 6 mostram que a tensão gerada é diretamente promocional à rotação, no entanto, a velocidade aumenta e a tensão também aumenta.
Podemos notar que quando aumenta a velocidade do vento, também o seu valor de tensão é aumentado.

\section{Medição em curto-circuito}

A curva curto-circuito também foi construída através da Fórmula 1, corrente versus RPM e medidos durante a operação do aerogerador, conforme Figura 7.

Os dados mostram que quanto maior for a rotação, maior vai ser sua corrente gerada, conforme mostra a Figura 7.

\section{Verificação de curva de potência}

A curva de potên também foi construída atrayés dos álcu de tensão e corrente con e a córm a 5, para geração da Fì 8, calculada a potência gera en ersas rotações com os dados med su vite a operação do aeroge

A. ra 8 mostra uma curva de o êncra tor cuída através da potência calc a a partir da multiplicação dos dau di tensão x corrente obtidos em nediçocs da Figura 7, e da velocidade do (RPM). Pode-se perceber, através do ráfico, que a relação entre potência e rotação são quase lineares. A curva gerada é muito semelhante à curva da tensão em função da velocidade do vento, visto que a tensão permanece praticamente constante. Com ajuda da bancada, podemos simular a ação do vento através da rotação do motor elétrico.

A incerteza para os valores calculados foi determinada, e a sua influência sobre os resultados é bastante pequena (da ordem de $\mathrm{mW}$ ).

A Figura 9 mostra as curvas que expressam à corrente convertida segundo 0 fabricante (calculada através dos dados medidos). Através destas curvas, pode-se observar que os valores de potência fornecidos pela curva do fabricante são bem próximos dos valores de potência verificados na prática para as mesmas velocidades do vento. 


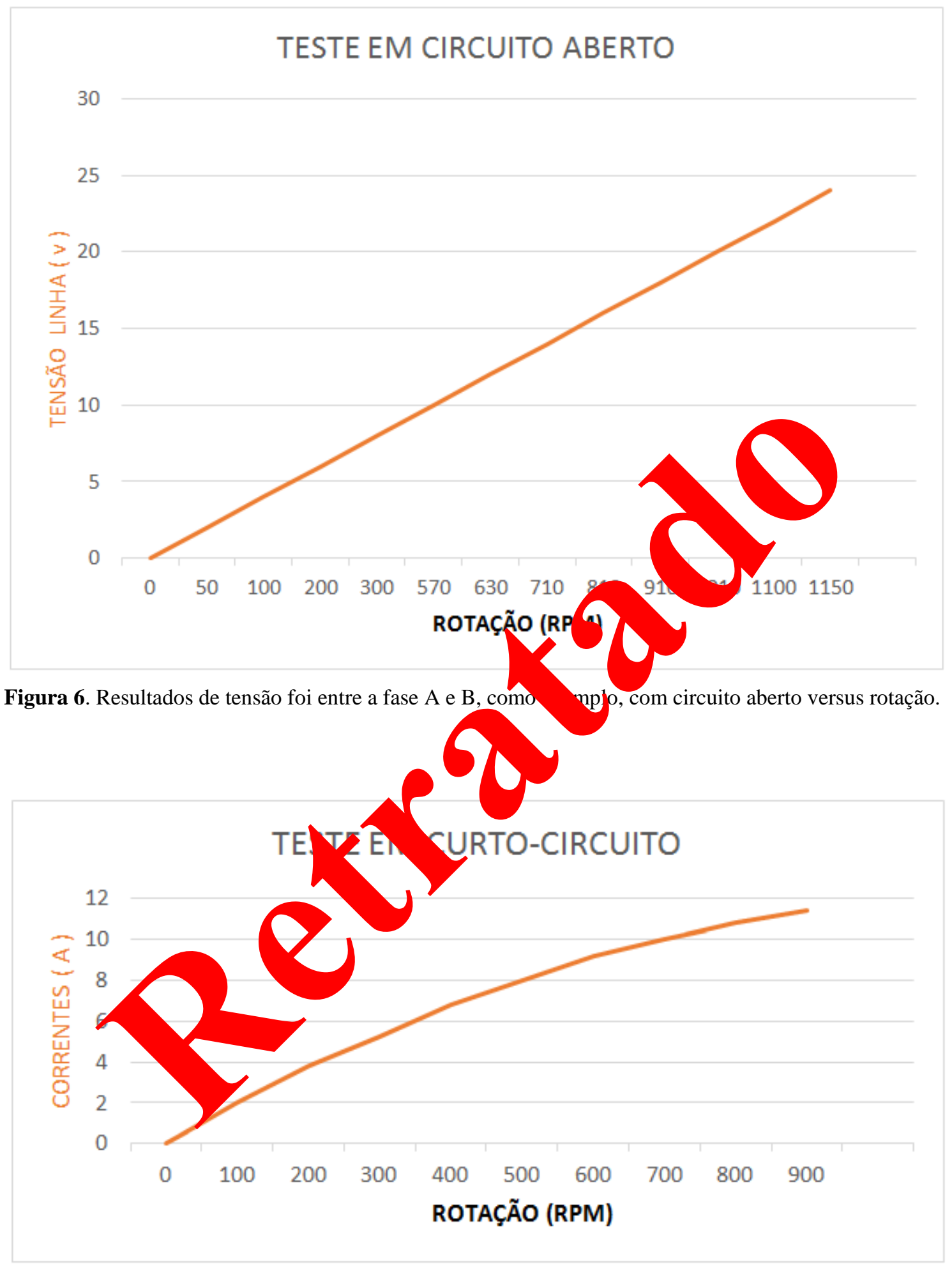

Figura 7. Resultados de corrente em curto-circuito, corrente versus rotação. 


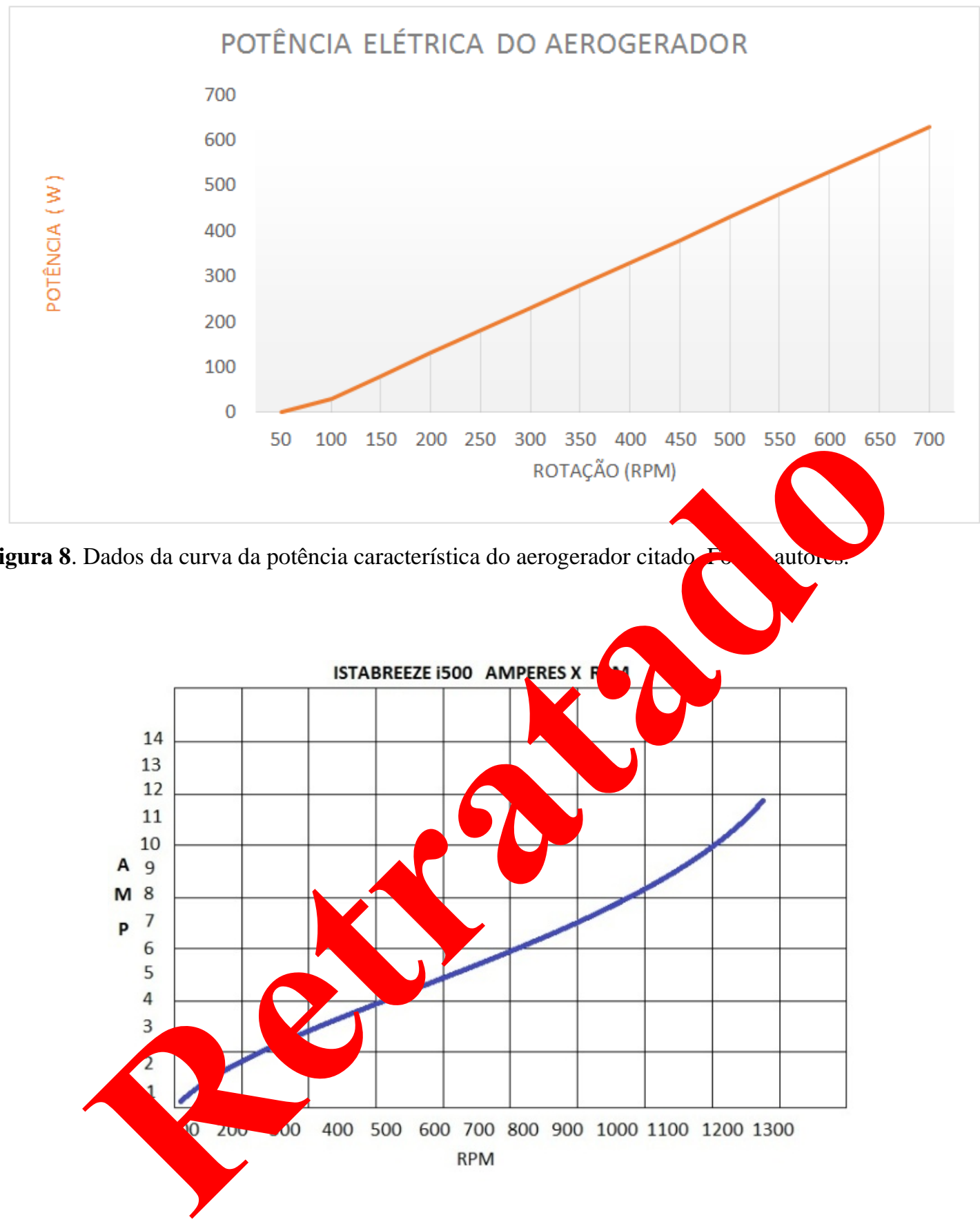

Figura 9. Dados da curva da corrente do aerogerador. Fonte: Altinel Energy Turquia - Fabricante.

\section{Conclusão}

Os resultados obtidos foram realizados em uma bancada, na qual foram levantados dados através dos três testes apresentados: tensões de saída do aerogerador em circuito aberto; medição de corrente em curto-circuito e verificação de curva de potência segundo IEC 61400-12-1; especificamente para um aerogerador de pequeno porte. Desse modo, a partir dos testes, temos parâmetros suficientes para analisar as características que os fabricantes informarem em seus manuais, podendo utilizar qualquer tipo de aerogerador de pequeno porte para analisar a viabilidade técnica de sua implantação no sistema de energia elétrica renovável. 


\section{Declaração de conflito de interesses}

Os autores declaram não haver conflitos de interesses.

\section{Referências}

ANEEL - Agência Nacional de Energia Elétrica. Resolução Normativa $n^{\circ}$ 482, de 17 de abril de 2012. Estabelece as condições gerais para o acesso de microgeração e minigeração distribuída aos sistemas de distribuição de energia elétrica, o sistema de compensação de energia elétrica, e dá outras providências. Disponível em: <http://www2.aneel.gov.br/ cedoc/ren2012482.pdf $>$. Acesso em: 28 nov. 2016.

Brum, T. S. Projeto de uso de energia fotovoltaica como fonte emergencial. Rio de Janeiro: Universidade Federal do Rio de Janeiro, 2012. (Trabalho de conclusão de curso de graduação). Disponível em: $<$ http://monografias.poli.ufrj.br/monografias/mo nopoli10006178.pdf>. Acesso em: 28 out. 2016.

Costa, G. B. Análise espacial e temporal do vento no Estado de Alagoas. Maceió: Universidade Federal de Alagoas, 2009. (Dissertação de mestrado).

Costa, G. B.; Lyra, R. F. F. Análise dos padrõos de vento no Estado de Alagoas. Rev ou Brasileira de Meteorologia, v. 27, n. 1, 138, 2012.

Ferreira Junior, J. C. G.; Rodrigu(a, G. Un estudo sobre a energia eólica po-Rrasi 'ôn cia Atual, v. 5, n. 1, p. 2-13, 20 5. sponive em: $<$ http://inseer.ibict bussj/in .php/dafsj/articl e/view/100/pdf . Ac em: $\triangle$ - 016.

Lopes, T. Aly sida J.A. Análise de dados da velo potencial eólico na Cidade do Grande. Anais do II Congresso Naciona Éngenharia Mecânica, João Pessoa, 2003. (C)-rom).
Martins, F. R.; Guarnieri, R. A.; Pereira, E. B. O aproveitamento da energia eólica. Rev. Brasil. Ens. Fís., v. 30, n. 1, p. 1304-1-1314-13, 2008. http://dx.doi.org/10.1590/S180611172008000100005

Matos, M. B. Investimentos financeiros em células a combustível e hidrogênio no Brasil. Campinas: Faculdade de Engenharia Mecânica, Universidade Estadual de Campinas, 2009. (Dissertação de mestrado).

Neoenergia. Geração de Energia no Brasil. $2009 . \quad$ Disponível em: $<$ http://www.neoenergia.com/section/geracao.as p>. Acesso em: 28 out. 2016.

Pessoa Júnior, O. Modelo causal dos primórdios da ciência do magnetismo Scientiae Studia, v. 8, n. 2, p. 195-212, 201

Picolo, A. P.; er, A Ra inelli, G. A. Uma abordagem a rólica como alternativa de ensi de tópicos de física clássica. Revi Brà ra de Ensino de Física, y 36, D. 306-1-4306-13, 2014. http:/d.au g/10.roso/S1806-

11172 \%0 0707

'S, L. I Feração de energia elétrica. b. ri: Marofle, 2015.

Salìn J. Energia eólica no Brasil: uma mparação do PROINFA e dos novos leilões. de Janeiro: Universidade Federal do Rio de aneiro, 2011. (Trabalho de conclusão de curso de graduação).

Vieira, J. C. S. F. Planejamento de cenários adotando a metodologia Shell no setor de energia elétrica: visualizações da geração distribuída no Brasil. 2013.

Informação da Licença: Este é um artigo Open Access distribuído sob os termos da Licença Creative Commons Attribution, que permite uso irrestrito, distribuição e reprodução em qualquer meio, desde que a obra original seja devidamente citada. 